\section{Autopercepção da saúde bucal de idosos de um município de médio porte do Nordeste brasileiro}

\author{
Self-perceived oral health among elderly \\ individuals in a medium-sized city in \\ Northeast Brazil
}

\author{
${ }^{1}$ Centro de Ciências da \\ Saúde, Universidade Federal \\ do Piauí, Teresina, Brasil. \\ Correspondência \\ L. C. A. Vasconcelos \\ Centro de Ciências da Saúde, \\ Universidade Federal do Piauí. \\ Av. Nossa Senhora de Fátima \\ 626, Parnaíba, PI 64202-220, \\ Brasil. \\ lu_correia26@hotmail.com
}

\section{Abstract}

This study focused on self-perceived oral health of the elderly and associated variables. The sample consisted of 321 functionally independent individuals 60 years or older living in Parnaíba, Piauí State, Brazil. Oral examinations were performed and questionnaires were applied during home visits. The elderly presented poor oral health, with mean DMFT 29.41 (SD = 4.10). However, their self-perceived oral health was positive, with $52 \%$ showing high scores according to the Geriatric Oral Health Assessment Index (GOHAI). Multivariate analysis showed that predictors of self-perceived oral health included the need for upper prostheses, oral mucosal lesions, and selfrated oral health. The most important predictor was self-rated oral health, with a weight of $20 \%$ variation in self-perceived oral health. In conclusion, subjective measurement of oral health in the elderly is less associated with their actual clinical status and more with other subjective factors.

Health of the Elderly; Oral Health; Quality of Life
Luciana Correia Aragão de Vasconcelos 1 Raimundo Rosendo Prado Júnior 1 João Batista Mendes Teles 1 Regina Ferraz Mendes 1

\section{Introdução}

A definição de saúde da Organização Mundial da Saúde (OMS) como o completo bem-estar físico, mental e social, e não apenas a ausência de doenças e enfermidades, é bastante discutida por alguns pesquisadores, que mais recentemente preferem o conceito de saúde relacionado à qualidade de vida 1 .

Com a mudança do paradigma biomédico, percebeu-se que aquela definição de saúde não continha alguns aspectos importantes da vida dos indivíduos. Dessa maneira, Locker 2 (p. 15) definiu a saúde como a "experiência subjetiva de uma pessoa em relação ao seu bem-estar funcional, social e psicológico", ou seja, refere-se à experiência individual e suas consequências na vida diária, constituindo-se em um conceito sociológico e psicológico, que pode ser aplicado a indivíduos ou a populações.

Segundo o mesmo autor, essa definição pertence ao paradigma socioambiental e cria um compromisso com o desenvolvimento de maneiras de medir percepções, sentimentos e comportamentos.

Tradicionalmente, as pesquisas relacionadas ao diagnóstico das condições de saúde das populações têm sido realizadas apenas por indicadores numéricos, e a determinação das necessidades apenas do ponto de vista normativo, ignorando os aspectos sociocomportamentais 
que deveriam ser considerados ${ }^{3}$. Segundo Gift \& Atchison ${ }^{4}$, historicamente, a maioria das investigações tem focado nos resultados físicos das doenças, como medidas de mortalidade e morbidade.

No entanto, os indicadores epidemiológicos são limitados a predizer o quanto determinada doença afeta a capacidade de um indivíduo desempenhar suas funções e atividades. Medidas de autopercepção dizem mais como está sendo afetada a rotina diária do indivíduo e da população em geral do que as realizadas com base em um ambiente clínico 5 .

O termo qualidade de vida relacionada à saúde bucal vem sendo usado por pesquisadores para reconhecer o impacto das doenças bucais na vida cotidiana das pessoas. Representa o fruto de muitas pesquisas e observações sobre o efeito que as desordens orais podem ter na qualidade de vida dos indivíduos. Tal noção apareceu somente no início dos anos 80 em contraste com a noção de qualidade de vida relacionada à saúde geral que emergiu no final dos anos 606 .

Gift \& Atchison 4 afirmaram que a qualidade de vida relacionada à saúde geral é um complexo multidimensional que incorpora domínios interrelacionados: (i) sobrevivência (por exemplo, ausência de câncer bucal, presença de dentes); (ii) a ausência de prejuízo, doença ou sintomas; (iii) funcionamento físico adequado associado com a mastigação, deglutição e ausência de desconforto e dor; (iv) funcionamento emocional associado com o sorriso; (v) funcionamento social associado com desempenho normal de papéis; (vi) as percepções de excelente saúde bucal; (vii) satisfação com a saúde oral; e (viii) ausência de desvantagem social ou cultural por causa da saúde bucal.

Reconhecendo, portanto, a importância da qualidade de vida relacionada à saúde bucal, pesquisadores desenvolveram questionários padronizados a fim de assimilá-la. Esses instrumentos foram elaborados para complementar os indicadores clínicos, buscando a coleta de dados sobre autopercepção, individual ou coletivamente, e de maneira que poderiam ser utilizados em programas educativos, preventivos e curativos e até mesmo por outros profissionais de saúde 7 .

Gift et al. ${ }^{8}$ propuseram um modelo conceitual teórico baseado em modelos sociológicos de interação para compreender a percepção global da saúde bucal. Os autores conjecturaram que a autopercepção da saúde oral é uma função de múltiplos fatores, incluindo características demográficas individuais, como idade e raça, fatores de predisposição e facilitação, tais como escolaridade, renda, autopercepção da saúde geral e orientações recebidas, fatores da condição de saúde bucal e fatores de autopercepção da necessidade de tratamento.

Entre os idosos, o valor dado a medições subjetivas se torna bastante importante, considerando que os problemas orais podem ter repercussões nutricionais e psicossociais 9 . Logo, para se diagnosticar as principais necessidades bucais da população idosa é necessário o conhecimento não só de suas necessidades objetivas (clínicas), mas também das subjetivas (relatadas) 10 .

Em 1990, Atchison \& Dolan 11 desenvolveram o Geriatric Oral Health Assessment Index (GOHAI), um índice de percepção do impacto da saúde bucal na rotina das pessoas idosas. $\mathrm{Na}$ construção desse índice, os autores entenderam a saúde bucal como a ausência de dor e infecção, consistindo numa dentição confortável e funcional (natural ou protética) que possibilita o indivíduo exercer seu papel na sociedade. Assim, criaram 12 itens que refletissem os problemas desses indivíduos em três dimensões: física, incluindo mastigação, dicção e deglutição; psicossocial, incluindo preocupação com a saúde oral, autoimagem, fuga dos contatos sociais e dor/ desconforto 11.

Investigações a respeito da autopercepção da qualidade de vida relacionada à saúde bucal têm sido desenvolvidas especialmente em países desenvolvidos como Estados Unidos 11,12,13, Inglaterra 14; Austrália 15,16,17 e Canadá 18,19,20,21. No Brasil, algumas investigações acerca do tema foram conduzidas, apesar disso existe ainda a necessidade de mais pesquisas para melhor compreensão dos fatores associados com a autopercepção da saúde bucal em nossa cultura $5,7,9,22,23,24,25,26,27,28,29,30,31,32$. Resultados desses estudos têm demonstrado a associação da autopercepção da saúde bucal com variáveis sociodemográficas 5,9,25,26,29,31, de saúde geral, de saúde bucal 5,7,9,22,25,27, de comportamento relacionado à saúde bucal 23 e a questões subjetivas 28,30 .

Tendo em conta que a coleta de informações sobre a autopercepção deve ser o primeiro passo para a formulação de políticas e programas odontológicos voltados à promoção, prevenção e recuperação da saúde bucal dos idosos 7, o objetivo deste estudo foi identificar a autopercepção da saúde bucal dos idosos de um município brasileiro de médio porte, por meio do indicador subjetivo GOHAI, e investigar as variáveis associadas a essa autopercepção.

\section{Métodos}

O estudo foi desenvolvido no Município de Parnaíba, localizado no extremo norte do Estado do Piauí, distante $339 \mathrm{~km}$ da capital, Teresina. Com 
145.729 habitantes de acordo com o censo de 2010 (http://www.ibge.gov.br, acessado em 16/ Jan/2011), é a segunda cidade mais populosa do estado.

A população para o estudo compreendeu os idosos com 60 anos ou mais de idade, de ambos os gêneros, cadastrados nas Equipes de Saúde da Família da zona urbana de Parnaíba.

De acordo com uma consulta feita ao Sistema de Informação da Atenção Básica (SIAB), no mês de março de 2009, quando da oportunidade do dimensionamento da amostra, estavam cadastradas 13.221 pessoas com 60 anos ou mais de idade no Estratégia Saúde da Família (ESF) na zona urbana de Parnaíba. Esse dado foi utilizado para o cálculo da amostra que permitisse estimativas com margem de erro de $\pm 5,54 \%$ e intervalo de 95\% de confiança (IC95\%). Desse modo, deveriam ser pesquisados 320 idosos dentre os indivíduos cadastrados nas equipes da zona urbana.

A pesquisa foi realizada em conformidade com a Resolução $n^{\circ}$. 196/1996 sobre pesquisa envolvendo seres humanos do Conselho Nacional de Saúde do Ministério da Saúde. O projeto de pesquisa foi submetido ao Comitê de Ética em Pesquisa (CEP) da Universidade Federal do Piauí, recebendo parecer favorável à sua realização (CAAE $n^{\circ}$. 0204.0.045.000-09). Todos os idosos participantes do estudo assinaram o Termo de Consentimento Livre e Esclarecido.

A amostragem foi probabilística em duas fases. Na primeira sessão, foram selecionadas por conveniência cinco equipes, $20,8 \%$ do total ( $\mathrm{n}=$ 24) de equipes que atuam na zona urbana. O sorteio das equipes foi feito por meio do uso de uma tabela de números aleatórios. Na segunda etapa, de acordo com o número de idosos cadastrados em cada equipe selecionada, verificou-se que para obtenção da amostra de maneira proporcional, seria necessário o sorteio de $15 \%$ dos idosos de cada equipe. Nessa etapa, foram utilizadas listas elaboradas no programa Excel (Microsoft Corp.) com os nomes dos idosos, por ordem alfabética, valendo-se do cadastro dos agentes comunitários de saúde (ACS). Para cada equipe sorteada, elaborou-se uma lista com os nomes e endereços dos idosos.

A escolha do idoso, então, deu-se por amostragem sistemática, sendo o primeiro escolhido ao acaso entre os nomes que ocupavam a 1a e a $6 \underline{a}$ posições na listagem, e assim a cada seis idosos listados, outro era sorteado. O intervalo de escolha foi determinado pela relação entre o número de idosos e o tamanho da amostra (15\%) calculada para a equipe.

Foram incluídos no estudo os idosos com 60 anos ou mais de idade, funcionalmente independentes e que tinham condições cognitivas para compreender as questões do formulário de pesquisa.

Um estudo piloto foi executado antes da coleta dos dados com $10 \%$ da amostra, ou seja, 32 idosos cadastrados em uma das Equipes de Saúde da Família sorteados para esse objetivo. Nessa fase, aplicou-se aos idosos sorteados um formulário de pesquisa inicial com o objetivo de ajustálo, verificando-se a necessidade de adequação de algumas de suas questões conforme a realidade encontrada. Além disso, foram realizados exames clínicos nos idosos, os quais foram repetidos após 20 dias, para verificação da concordância intraexaminadora pelo índice kappa.

$\mathrm{O}$ resultado do índice kappa foi de 0,993 $(\mathrm{p}=0,00)$ para o CPOD; $0,760(\mathrm{p}=0,000)$ para necessidade de prótese superior; $0,914(\mathrm{p}=0,000)$ para necessidade de prótese inferior e para alterações em tecido mole, o índice kappa foi de $0,655(\mathrm{p}=0,000)$.

A coleta dos dados foi realizada em visitas domiciliares por uma cirurgiã-dentista acompanhada de uma técnica em saúde bucal, no período de março a julho de 2010, os dados foram coletados por meio da aplicação de um formulário de pesquisa e pela realização de exames clínicos bucais.

A examinadora utilizou para realização dos exames espelho bucal plano, sonda periodontal milimetrada preconizada pela OMS, espátula de madeira, gaze e iluminação artificial de coloração azul-branca (lanterna de led), como indicado pela OMS 33 .

Destaca-se que as visitas eram agendadas previamente pelos ACS. No entanto, se o idoso não era encontrado por duas vezes na residência, novo indivíduo era sorteado. Assim, podiam-se compensar as perdas da amostra. Apenas um idoso se recusou a participar da pesquisa.

O formulário relativo à autopercepção da qualidade de vida relacionada à saúde bucal era composto por 12 questões baseadas no GOHAI 11.

O GOHAI utilizado neste estudo foi o modificado, com apenas três opções de respostas, o mesmo já usado por outros pesquisadores 9,10,13,23,34,35,36 e relatado por Atchison 37, em 1997. As cinco alternativas de respostas do índice original foram agrupadas em três: "sempre", “às vezes", "nunca”, nesse caso o escore do indivíduo poderia variar de 12-36, e quanto mais alto o valor, melhor autopercepção da saúde bucal e qualidade de vida do indivíduo. A despeito dessa modificação e fazendo uma adaptação do índice original, escores do GOHAI entre 34-36 são considerados altos, entre 31-33 são considerados moderados, e valores menores ou iguais a 30 são baixos. 
O exame clínico das condições dentárias, necessidade de próteses e presença de alterações em tecido mole foram executados conforme os critérios descritos no manual do examinador do Projeto SBBrasil do Ministério da Saúde 38 . Importante destacar que o referido manual não menciona os critérios para necessidade de prótese, por conseguinte, a necessidade de substituições não foi escopo desta pesquisa, procurou-se apenas identificar se no momento do exame o paciente fazia uso das próteses ou se necessitava das mesmas.

A variável dependente escolhida para o estudo foi o índice GOHAI. Utilizou-se a sua classificação pelos critérios de pontuação em "baixo", "moderado" e "alto" como observado em algumas pesquisas 9,11,35. Porém, para análise dos dados, utilizou-se a mediana para dicotomizar a classificação, de maneira que os que obtiveram pontuação do GOHAI menor que 34 (baixo/ moderado) formaram um grupo e os que pontuaram acima de 34 (alto) formaram um segundo grupo.

As variáveis independentes foram reunidas em 4 grupos, baseadas no modelo de Gift et al. 8, com algumas modificações: (i) variáveis demográficas: gênero (feminino, masculino) e faixa etária (60-70, 70-80, maior que 80); (ii) variáveis de predisposição e facilitação: escolaridade $(\leq 5$ anos, de 6-10 anos, $\geq 11$ anos); classe econômica (classe A/B, classe C, classe $\mathrm{D} / \mathrm{E}$ ); autoavaliação da saúde geral (excelente/boa, regular, ruim/não sabe) e última visita ao cirurgião-dentista (nunca foi/há menos de 1 ano/de 1-2 anos/de 2-5 anos/ há mais de 5 anos); (iii) variáveis clínicas de saúde bucal: edentulismo (sim, não); índice CPOD (média); necessidade de tratamento protético superior e inferior (não necessita, necessita uma prótese parcial, necessita uma combinação de próteses, necessita prótese dentária total); alteração em tecido mole (sim, não); (iv) variável de autoavaliação da saúde bucal (excelente/boa, regular, ruim/não sabe).

$\mathrm{Na}$ caracterização da classe econômica, foi aplicado o Critério de Classificação Econômica Brasil 2008 (CCEB2008) da Associação Brasileira de Empresas de Pesquisas (ABEP) 39.

Para verificar diferenças com significância estatística entre os grupos da variável dependente classificação GOHAI, foram utilizados os testes não paramétricos Mann-Whitney e o teste qui-quadrado de Pearson. Em todos os testes, o $\alpha$ foi fixado em $5 \%$. Análise de regressão linear multivariada foi realizada para verificação dos preditores da classificação GOHAI, com as variáveis entrando em 4 blocos de acordo com os grupos de fatores do modelo teórico de Gift et al. 8. Todos os dados foram analisados com uso do programa SPSS, versão 16.0 (SPSS Inc., Chicago, Estados Unidos).

\section{Resultados}

Participaram da pesquisa 321 idosos, de ambos os gêneros, funcionalmente independentes, residentes na zona urbana do Município de Parnaíba e cadastrados na ESF.

Dos entrevistados, 119 (37,1\%) eram do gênero masculino e 202 (62,9\%) eram do gênero feminino. Os achados mostraram uma maior participação de indivíduos na faixa etária entre 60-70 anos de idade (48,3\%), com menos de cinco anos de escolaridade $(86,3 \%)$, e pertencentes à classe econômica D e E (54,5\%).

O CPOD médio observado para a amostra total deste estudo foi de 29,41 (DP = 4,10), sendo o componente perdido responsável por $92,16 \%$ desse índice. Quanto ao número de dentes remanescentes, encontrou-se que somente $6,2 \%$ dos idosos apresentavam 20 dentes ou mais. Quanto à prevalência de edentulismo, observouse que $52 \%$ dos idosos já haviam perdido todos os dentes.

A necessidade de prótese superior foi percebida em $34,3 \%$ dos idosos, dentre os quais $21,5 \%$ referiam-se à prótese total. Para necessidade de prótese inferior, o resultado foi de $62,6 \%$ dos indivíduos necessitando de reabilitação na arcada, dos quais $33,3 \%$ referiam-se à prótese total. Ainda de acordo com o exame epidemiológico, $34,3 \%$ dos idosos apresentaram alguma alteração em tecido mole.

$\mathrm{Na}$ análise dos dados subjetivos, verificouse que a média GOHAI obtida foi de 32,59 (DP = $3,44)$, sendo classificada como moderada de acordo com os critérios desse índice. Contudo, considerou-se que a autopercepção foi positiva, já que $52 \%$ dos indivíduos tiveram escore alto de acordo com a classificação do GOHAI. Quando questionados sobre a condição de saúde bucal, por uma única questão, 47\% dos idosos responderam excelente ou boa.

Na associação entre a variável dependente classificação GOHAI, dividida em dois grupos (baixo/moderado e alto) com as variáveis independentes, verificou-se uma associação significativa com o edentulismo, alteração em tecido mole e com a autoavaliação da saúde bucal. A Tabela 1 mostra, para cada variável independente, os valores percentuais da classe mais expressiva.

A análise de regressão multivariada foi realizada a fim de investigar os preditores da classificação GOHAI. As variáveis independentes entraram no modelo em 4 etapas, como mostrado na Tabela 2, de acordo com o modelo teórico 
Distribuição da autopercepção da saúde bucal dos idosos medida pelo Geriatric Oral Health Assessment Index (GOHAl) de acordo com as classes mais expressivas das variáveis do estudo. Parnaíba, Piauí, Brasil, março a julho de 2010.

\begin{tabular}{lccc}
\hline Variáveis & Baixo/Moderado & Alto & Valor de p \\
\hline Gênero (\% feminino) & 45,5 & 54,5 & 0,15 \\
Faixa etária (\% 60-70 anos) & 52,3 & 47,7 & 0,10 \\
Escolaridade (\% 5 anos ou menos) & 46,9 & 53,1 & 0,62 \\
Classe econômica (\% classe D/E) & 48,0 & 49,0 & 0,59 \\
Autoavaliação da saúde geral (\% regular) & 50,6 & 58,8 & 0,08 \\
Última visita ao consultório dentário (\% nunca foi) & 41,2 & 62,3 & 0,12 \\
Edentulismo (\% sim) & 37,7 & 29,5 & 0,82 \\
CPOD (média) & 29,2 & 54,5 & 0,14 \\
Necessidade de prótese superior (\% não necessitam) & 45,5 & 57,5 & 0,38 \\
Necessidade de prótese inferior (\% não necessitam) & 42,5 & 46,4 & 0,00 * \\
Alteração em tecido mole (\% não) & 53,6 & 23,8 & \\
Autoavaliação da saúde bucal (\% excelente/boa) & & 5,2 & \\
\hline
\end{tabular}

* Estatisticamente significativo.

escolhido para o estudo. Na primeira etapa, as variáveis demográficas foram responsáveis por uma variância residual (R2) de 0,128 , ou seja, explicavam apenas $12,8 \%$ da variação da classificação GOHAI.

Quando as variáveis de predisposição e facilitação foram incluídas no modelo, a variância residual $\left(\mathrm{R}^{2}\right)$ passou para 0,205. Após a entrada das variáveis clínicas, a variância residual $\left(\mathrm{R}^{2}\right)$ aumentou para 0,308 , fazendo concluir que essas eram responsáveis por apenas 10 \% da variação da autopercepção medida pelo GOHAI.

Na última etapa, com a inclusão da variável independente autoavaliação da saúde bucal, a variância residual ( $\left.\mathrm{R}^{2}\right)$ foi de 0,516 , mostrando que $20 \%$ da variação do GOHAI podiam ser explicadas por essa variável. Os fatores significativos associados ao GOHAI no modelo final foram a necessidade de prótese superior, alteração em tecido mole e a autoavaliação da saúde bucal.

\section{Discussão}

Considerando que a ESF no Município da Parnaíba possuía cobertura estimada de $83,34 \% 40$ em março de 2009 e que foram selecionados os indivíduos cadastrados apenas na zona urbana, a amostra foi, portanto, representativa da população urbana coberta pela ESF de Parnaíba.

As limitações do estudo dizem respeito a uma amostra que se constituiu em sua maioria de mulheres, de indivíduos pertencentes à classe econômica baixa, e que possuíam pouca ou nenhuma escolaridade, características que demonstram a realidade de vida dos idosos no país. Como benefício para a pesquisa, participaram indivíduos independentes, moradores de seus domicílios e, por conseguinte, com menor interferência de comorbidades, mais comuns em indivíduos institucionalizados e/ou com alto grau de dependência ${ }^{9}$.

O levantamento dos dados clínicos mostrou um quadro de precárias condições de saúde bucal, com um grande número de dentes perdidos e uma porcentagem alta de edentulismo. O CPOD encontrado foi bastante elevado, com um peso maior para os dentes perdidos, como visto em outras investigações sobre saúde bucal dos idosos 9,23,31,41,42,43.

Quanto à autopercepção do impacto das condições de saúde bucal na qualidade de vida, utilizou-se o indicador GOHAI. Como lembram Atchison \& Dolan 11, o GOHAI é um instrumento de avaliação e não uma medida objetiva de saúde bucal, consequentemente, o GOHAI não pode ser usado para diagnosticar doenças bucais. Todavia, em nível individual, por exemplo, o seu uso por outros profissionais como um geriatra pode indicar a necessidade de um exame clínico bucal; e em nível populacional, seria um meio de coleta de informações sobre problemas bucais, ou mesmo uma forma de avaliação dos resultados dos tratamentos odontológicos.

A média de tal índice neste estudo foi de 32,59 (DP $=3,44$ ), resultado muito próximo do obtido por Mesas et al. 9, que observaram um índice do GOHAI médio de 33,03 para idosos 
Resultados da regressão linear multivariada dos idosos segundo as variáveis demográficas, de predisposição e facilitação, clínicas e de autoavaliação da saúde bucal. Variável dependente: classificação do Geriatric Oral Health Assessment Index (GOHAl). Parnaíba, Piauí, Brasil, março a julho de 2010.

\begin{tabular}{|c|c|c|c|c|c|c|c|c|}
\hline \multirow[t]{3}{*}{ Variáveis independentes } & \multicolumn{8}{|c|}{ Etapas } \\
\hline & \multicolumn{2}{|c|}{ Primeira } & \multicolumn{2}{|c|}{ Segunda } & \multicolumn{2}{|c|}{ Terceira } & \multicolumn{2}{|c|}{ Quarta } \\
\hline & $\boldsymbol{\beta}$ & Valor de p & $\beta$ & Valor de p & $\beta$ & Valor de $p$ & $\beta$ & Valor de $p$ \\
\hline \multicolumn{9}{|l|}{ Demográficas } \\
\hline Gênero & 0,062 & 0,27 & 0,070 & 0,21 & 0,048 & 0,39 & 0,061 & 0,23 \\
\hline Faixa etária & 0,111 & 0,04 * & 0,133 & 0,02 * & 0,102 & 0,07 & 0,087 & 0,09 \\
\hline \multicolumn{9}{|l|}{ Predisposição e facilitação } \\
\hline Escolaridade & & & $-0,007$ & 0,91 & 0,022 & 0,73 & 0,010 & 0,87 \\
\hline Classe econômica & & & 0,066 & 0,31 & 0,085 & 0,19 & 0,038 & 0,52 \\
\hline Autoavaliação saúde geral & & & $-0,150$ & 0,00 * & $-0,131$ & 0,01 * & $-0,065$ & 0,20 \\
\hline Última visita ao consultório dentário & & & $-0,028$ & 0,61 & $-0,017$ & 0,75 & $-0,24$ & 0,62 \\
\hline \multicolumn{9}{|l|}{ Clínicas } \\
\hline Edentulismo & & & & & 0,222 & 0,00 * & 0,076 & 0,22 \\
\hline CPOD & & & & & $-0,122$ & 0,05 & $-0,083$ & 0,15 \\
\hline Necessidade de prótese superior & & & & & 0,059 & 0,37 & 0,125 & 0,04 * \\
\hline Necessidade de prótese inferior & & & & & $-0,065$ & 0,28 & $-0,043$ & 0,43 \\
\hline Alteração de tecido mole & & & & & 0,094 & 0,13 & 0,110 & 0,05 \\
\hline Autoavaliação saúde bucal & & & & & & & $-0,451$ & $0,00 *$ \\
\hline $\mathrm{R}^{2}$ & \multicolumn{2}{|r|}{0,128} & \multicolumn{2}{|c|}{0,205} & \multicolumn{2}{|c|}{0,308} & \multicolumn{2}{|c|}{0,516} \\
\hline
\end{tabular}

* Estatisticamente significativo.

independentes; Silva \& Fernandes 7 que encontraram um GOHAI de 33,80 para indivíduos dentados; e de Silva et al. 23 que verificaram um índice de 33,61 para idosos conveniados ou não a planos de saúde odontológicos. Pelo critério de classificação por meio de pontuação do GOHAI, $52 \%$ dos idosos tiveram escores classificados como altos, demonstrando um resultado positivo, apesar da pobre situação de saúde bucal.

Questiona-se, consequentemente, como a autopercepção dos idosos foi positiva em condições odontológicas tão precárias. Isso, contudo, também foi relatado por outros autores 9,23,34. De acordo com Silva ${ }^{34}$, talvez as medidas clínicas de saúde utilizadas pelo profissional sejam preditores relativamente fracos da percepção de saúde bucal das pessoas. Resultados obtidos por Leão \& Sheiham 14 confirmaram que existe uma significativa, mas fraca relação entre o estado de saúde bucal e medidas subjetivas.

Para Nunes \& Abegg 27, os idosos percebem a sua saúde bucal fazendo uso, por exemplo, de sinais e sintomas tais como dor, ou de problemas que afetam a mastigação e interferem na aparência, diferentemente da avaliação realizada pelos profissionais. Além disso, a percepção da saúde bucal é influenciada por crenças e pelo valor atribuído à saúde perdida pelo indivíduo afetado 3 . Hiramatsu et al. 24 acreditam que, de modo especial, os idosos têm sua percepção afetada pela crença de que algumas dores e incapacidades são naturais do envelhecimento.

Mesmo com essa diferença entre as avaliações do profissional e do paciente, Locker et al. 18 defendem a utilização dos indicadores subjetivos em tempo de escassez de recursos para identificar subgrupos que necessitam de maior prioridade quanto aos cuidados de saúde bucal.

Entretanto, de acordo com Mesas et al. 9, o GOHAI não tem mostrado ser um bom instrumento no contexto brasileiro na identificação de indivíduos com saúde oral deficiente, mas para indivíduos que necessitam de cuidados multiprofissionais. Para esses autores, a análise de diferentes dimensões do GOHAI, em cada contexto, ao invés de usar o escore global, poderia fornecer informações mais específicas sobre as necessidades de cuidados bucais. Essa opinião vai de encontro à de Silva ${ }^{34}$, para quem a utilização do índice GOHAI pode ser relevante, uma vez que em seu estudo ele foi um preditor importante na avaliação da saúde bucal pelas pessoas. 
Apesar de algumas críticas relacionadas ao uso de indicadores subjetivos em pesquisas de avaliação da necessidade de tratamento odontológico, acredita-se na importância da utilização desses dados para melhor compreensão de comportamentos relacionados à saúde bucal. A coleta de informações desse tipo permite, por exemplo, o entendimento das atitudes dos indivíduos quanto ao autocuidado e à procura por serviços odontológicos.

$\mathrm{Na}$ busca de um melhor entendimento dos fatores relacionados à autopercepção da saúde bucal, usou-se neste estudo o modelo teórico proposto por Gift et al. 8, assim como realizado por outros autores 25,28,30. De acordo com a análise estatística, os fatores que mostraram associação com a classificação GOHAI da amostra global foram o edentulismo, alteração em tecido mole e a autoavaliação da saúde bucal.

Nenhuma das variáveis demográficas e de facilitação e predisposição mostraram-se associadas ao GOHAI. Na análise multivariada, somente a faixa etária foi significativa durante as etapas 1 e 2 do modelo, exatamente quando haviam sido consideradas apenas essas variáveis.

Kressin et al. 13 pesquisaram duas amostras de idosos e encontraram em uma delas diferenças significativas da média do GOHAI entre idosos de idades diferentes, em que os mais jovens tiveram uma melhor autopercepção da saúde bucal. Nessa mesma amostra, os indivíduos casados, brancos e com melhor nível educacional tiveram escores mais altos. A renda, porém, apresentou impacto nas médias de ambas as amostras, e nas pessoas com renda maior, o GOHAI foi mais alto.

No estudo de Silva \& Fernandes 7, a classe social se mostrou associada com a autoavaliação da saúde bucal. Na investigação de Bulgarelli \& Manço 29, os autores observaram que quanto maior a idade e menor a escolaridade, menor era o número de idosos insatisfeitos com a saúde bucal. Mesas et al. 9 encontraram associação entre a autopercepção negativa da saúde bucal e o gênero, o que não foi encontrado neste estudo.

As variáveis clínicas de saúde bucal associadas ao GOHAI neste estudo foram o edentulismo e alteração em tecido mole. Os edêntulos tiveram boa percepção da saúde bucal, como se percebe pelos resultados obtidos. No estudo de Benedetti et al. 26, os autores verificaram que a falta de dentes não é percebida pela maioria dos idosos como fator prejudicial à capacidade de mastigação, pois apenas $19,8 \%$ responderam ter comprometimento da mastigação por problemas bucais. Os autores acreditam que essa não percepção, provavelmente, deve-se à adaptação da alimentação à utilização de próteses, mesmo essa condição não permitindo uma mastigação satisfatória. Narvai \& Antunes 22 encontraram que o uso da prótese dentária total contribui significativamente para suavizar o grau de comprometimento das funções bucais, pois situações indesejáveis como "ficar insatisfeito ao ver os dentes no espelho" ou "sentir que os dentes não estão bem", acontecem expressivamente menos entre os que usam o aparelho protético total.

Para Martins et al. 30, os idosos viveram em um período em que a perda dos dentes e as condições ruins dos dentes, assim como o adoecimento, eram entendidas parte do processo normal do envelhecimento. Ainda de acordo com Benedetti et al. 26, a ausência de dentes não é vista como problema de saúde bucal pelos idosos. Para Matos \& Lima-Costa 25 , devido a problemas constantes com os dentes naturais, os idosos consideram haver uma melhoria da saúde bucal com a substituição deles por próteses.

No modelo final da análise multivariada com todos os fatores pesquisados, verificou-se que os preditores da autopercepção da saúde bucal foram necessidade de prótese superior, as alterações em tecido mole e a autoavaliação da saúde bucal. No entanto, as variáveis clínicas foram responsáveis por $10 \%$ da predição, enquanto a avaliação por um único item foi responsável por $20 \%$ da variação do GOHAI, demonstrando que tal variável teve maior importância na determinação da autopercepção da saúde bucal.

Outros estudos têm demonstrado uma associação entre as variáveis clínicas de saúde bucal e medidas subjetivas, mas a associação tem se mostrado sem força. Konishi et al. 44 realizaram um estudo para avaliar a relação entre o estado de saúde bucal, função motora oral, situações da vida diária e a autoavaliação da saúde bucal entre 190 idosos independentes da cidade de Tóquio, no Japão. Os achados revelaram que as situações da vida diária e função motora oral tiveram efeitos independentes na autoavaliação da saúde bucal e que a relação entre a autoavaliação da saúde bucal e as condições de saúde bucal era fraca.

Entre as variáveis clínicas preditoras da percepção neste estudo, observou-se a necessidade de prótese superior. No estudo de Biazevic et al. 5 , os autores observaram que não havia impacto na saúde bucal relacionado ao uso de prótese total, mas existia essa interferência associada à necessidade de prótese total superior e inferior. Nunes \& Abegg 27 encontraram em sua investigação que a dor de dente e a necessidade de próteses dentárias foram os principais fatores que afetaram a autopercepção da saúde bucal de idosos do sul do Brasil. 
Importante salientar que a prótese superior está bastante relacionada ao aspecto estético. Os idosos, em geral, consideram o uso da prótese superior mais importante do que a inferior, fato comprovado, na presente pesquisa, pela menor necessidade de reabilitação protética para a arcada superior.

Quanto à variável alteração em tecido mole, na investigação de Martins et al. 30, os autores observaram que alterações da mucosa estavam relacionadas à autoavaliação da saúde bucal, e os indivíduos que apresentaram lesões em tecido mole também apresentaram uma maior frequência de autoavaliação negativa. Nesta pesquisa, demonstrou-se também uma associação entre as variáveis alterações em tecido mole e autopercepção da saúde bucal, contudo os resultados encontrados foram discordantes, já que entre os que não possuíam alterações de mucosa, a autopercepção mostrou-se pior.

Segundo Martins et al. 28, a falta de associação entre a presença de alterações em tecidos moles e a autopercepção da necessidade de tratamento odontológico é motivo de preocupação, pois o reconhecimento dessas alterações é importante para a procura pelos serviços odontológicos e para o diagnóstico precoce do câncer de boca.

O fator preditor mais importante da autopercepção da saúde bucal, todavia, foi a autoavaliação da saúde bucal, que assim como a variável dependente é também uma variável subjetiva. Como relatado anteriormente, essa variável pode explicar $20 \%$ da variação do GOHAI. No estudo de Martins et al. ${ }^{32}$, os autores concluíram que condições subjetivas estavam mais fortemente associadas à autoavaliação da saúde bucal entre os idosos brasileiros. Outras investigações também demonstraram associação entre o GOHAI e autoavaliação da saúde bucal. Atchison \& Dolan 11 encontraram correlação significativa entre a autoavaliação da saúde bucal feita por apenas uma pergunta e o GOHAI. Kressin et al. 13 observaram que, além da renda, a autoavaliação da saúde bucal foi um preditor significativo para a média do GOHAI. Atieh 45 encontrou que os pacientes que perceberam o seu estado de saúde bucal como bom tinham maior probabilidade de ter um GOHAI significativamente maior comparados com aqueles que perceberam a saúde bucal como precária.

\section{Conclusão}

Ficaram evidentes as precárias condições de saúde bucal dos indivíduos pesquisados, com grande número de dentes perdidos. Ainda assim, verificou-se uma autopercepção positiva da saúde bucal. As variáveis associadas à autopercepção dos idosos foram o edentulismo, as alterações em tecido mole e a autoavaliação de saúde bucal. Resultados da análise multivariada demonstraram, no entanto, que os preditores da autopercepção foram necessidade de prótese superior, alterações em tecido mole e autoavaliação de saúde bucal. As variáveis clínicas foram responsáveis por apenas $10 \%$ da variação da autopercepção, e a variável preditora mais importante foi a autoavaliação de saúde bucal, com um peso de $20 \%$. A medida subjetiva relacionada à saúde bucal dos idosos está menos associada às situações clínicas que eles apresentam - possivelmente por falta de orientação e acesso aos serviços odontológicos - e mais ligada a fatores subjetivos. Recomenda-se, ainda, um maior número de estudos acerca do tema para formação de uma base conceitual mais densa nessa linha de pesquisa, com intuito de melhor compreendermos os fatores ligados à autopercepção da saúde bucal dos idosos e, consequentemente, dos comportamentos em relação à saúde bucal desses indivíduos. 


\section{Resumo}

Este estudo objetivou identificar a autopercepção da saúde bucal de idosos e investigar as variáveis associadas a essa autopercepção. Foram pesquisados 321 indivíduos, de 60 anos ou mais, funcionalmente independentes, residentes do Município de Parnaíba, Piauí, Brasil. Realizaram-se exames clínicos bucais e aplicaram-se formulários durante visitas domiciliares. Os idosos apresentaram precárias condições de saúde bucal, com média CPOD de 29,41, (DP=4,10). A autopercepção da saúde bucal, medida pelo Geriatric Oral Health Assessment Index (GOHAI), no entanto, foi positiva, 52\% dos idosos obtiveram escores altos no GOHAI. Resultados da análise multivariada demonstraram que os preditores da autopercepção foram necessidade de prótese superior, alterações em tecido mole e autoavaliação de saúde bucal. A preditora mais importante foi a autoavaliação de saúde bucal, com um peso de $20 \%$ na variação da autopercepção. Concluiu-se que a medida subjetiva relacionada à saúde bucal dos idosos está menos associada às situações clínicas que eles apresentam e mais ligada a outros fatores subjetivos.

Saúde do Idoso; Saúde Bucal; Qualidade de Vida

\section{Colaboradores}

L. C. A. Vasconcelos contribuiu substancialmente para a concepção e planejamento da pesquisa, análise e interpretação dos dados e elaboração do rascunho e aprovação final do manuscrito. R. F. Mendes contribuiu substancialmente para concepção e planejamento da pesquisa, na análise e interpretação dos dados, na revisão crítica do conteúdo e na aprovação final do manuscrito. R. R. Prado Júnior contribuiu substancialmente para a análise e interpretação dos dados, revisão crítica do conteúdo e aprovação final do manuscrito. J. B. M. Teles contribuiu substancialmente na análise e interpretação dos dados.

\section{Agradecimentos}

À Prefeitura Municipal de Parnaíba, Piauí.

\section{Referências}

1. Hebling E, Pereira AC. Oral health-related quality of life: a critical appraisal of assessment tools used in elderly people. Gerodontology 2007; 24:151-61.

2. Locker D. Concepts of oral health, disease and the quality of life. In: Slade GD, editor. Measuring oral health and quality of life. Chapel Hill: University of North Carolina, Dental Ecology; 1997. p. 11-23.

3. Reis SCGB, Marcelo VC. Saúde bucal na velhice: percepção dos idosos, Goiânia, 2005. Ciênc Saúde Coletiva 2006; 11:191-9.

4. Gift HC, Atchison KA. Oral health, health, and health-related quality of life. Med Care 1995; 33(11 Suppl):NS57-77.

5. Biazevic MGH, Michel-Crosato E, Iagher F, Pooter CE, Correa SL, Grasel CE. Impact of oral health on quality of life among the elderly population of Joaçaba, Santa Catarina, Brazil. Braz Oral Res 2004; 18:85-91.

6. Al Shamrany M. Oral health-related quality of life: a broader perspective. East Mediterr Health J 2006; 12:894-901.

7. Silva SRC, Fernandes RAC. Autopercepção das condições de saúde bucal por idosos. Rev Saúde Pública 2001; 35:349-55.
8. Gift HC, Atchison KA, Drury TF. Perceptions of the natural dentition in the context of multiple variables. J Dent Res 1998; 77:1529-38.

9. Mesas AE, Andrade SM, Cabrera MAS. Factors associated with negative self-perception of oral health among elderly people in a Brazilian community. Gerodontology 2008; 25:49-56.

10. Silva EFA, Sousa MLR. Autopercepção da saúde bucal e satisfação com a vida em mulheres idosas usuárias de prótese total. Rev Odontol Univ Cid Sao Paulo 2006; 18:61-5.

11. Atchison KA, Dolan TA. Development of the geriatric oral health assessment index. J Dent Educ 1990; 54:680-7.

12. Dolan TA. The sensitivity of the Geriatric Oral Health Assessment Index to dental care. J Dent Educ 1997; 61:37-46.

13. Kressin NR, Atchison KA, Miller DR. Comparing the impact of oral disease in two populations of older adults: application of the Geriatric Oral Health Assessment Index. J Public Health Dent 1997; 57:224-32. 
14. Leão A, Sheiham A. Relation between clinical dental status and subjective impacts on daily living. J Dent Res 1995; 74:1408-13.

15. Slade GD, Spencer AJ. Development and evaluation of the oral health impact profile. Community Dent Health 1994; 11:3-11.

16. Slade GD, Spencer AJ, Locker D, Hunt RJ, Strauss RP, Beck JD. Variations in the social impact of oral conditions among older adults in South Australia, Ontario, and North Carolina. J Dent Res 1996; 75:1439-50.

17. Slade GD. Derivation and validation of a shortform oral health impact profile. Commnunity Dent Oral Epidemiol 1997; 25:284-90.

18. Locker D, Jokovic A. Using subjective oral health status indicators to screen for dental care needs in older adults. Community Dent Oral Epidemiol 1996; 24:398-402.

19. Locker D, Jokovic A. Three-year changes in selfperceived oral health status in an older Canadian population. J Dent Res 1997; 76:1292-7.

20. Locker D, Clarke M, Payne B. Self-perceived oral health status, psychological well-being, and life satisfaction in an older adult population. J Dent Res 2000; 79:970-5.

21. Locker D, Gibson B. Discrepancies between selfratings of and satisfaction with oral health in two older adult populations. Community Dent Oral Epidemiol 2005; 33:280-8.

22. Narvai PC, Antunes JLF. Saúde bucal: a autopercepção da mutilação e das incapacidades. In: Lebrão ML, Duarte YAO, organizadores. SABE - Saúde, Bem-estar e Envelhecimento. O projeto SABE no Município de São Paulo: uma abordagem inicial. Brasília: Organização Pan-Americana da Saúde; 2003. p. 121-37.

23. Silva DD, Sousa MLR, Wada RS. Autopercepção e condições de saúde bucal em uma população de idosos. Cad Saúde Pública 2005; 21:1251-9.

24. Hiramatsu DA, Franco LJ, Tomita NE. Influência da aculturação na autopercepção dos idosos quanto à saúde bucal em uma população de origem japonesa. Cad Saúde Pública 2006; 22:2441-8.

25. Matos DL, Lima-Costa MF. Autoavaliação da saúde bucal entre adultos e idosos residentes na Região Sudeste: resultados do Projeto SB-Brasil, 2003. Cad Saúde Pública 2006; 22:1699-707.

26. Benedetti TRB, Mello ALSF, Gonçalves LHT. Idosos de Florianópolis: autopercepção das condições de saúde bucal e utilização de serviços odontológicos. Ciênc Saúde Coletiva 2007; 12:1683-90.

27. Nunes CIP, Abegg C. Factors associated with oral health perceptions in older Brazilians. Gerodontology 2008; 25:42-8.

28. Martins AMEBL, Barreto SM, Pordeus IA. Fatores relacionados à autopercepção da necessidade de tratamento odontológico entre idosos. Rev Saúde Pública 2008; 42:487-96.

29. Bulgarelli AF, Manço ARX. Idosos vivendo na comunidade e a satisfação com a própria saúde bucal. Ciênc Saúde Coletiva 2008; 13:1165-74.

30. Martins AMEBL, Barreto SM, Pordeus IA. Autoavaliação de saúde bucal em idosos: análise com base em modelo multidimensional. Cad Saúde Pública 2009; 25:421-35.
31. Sousa EHAS, Barbosa MBCB, Oliveira PAP, Espíndola J, Gonçalves KJ. Impacto da saúde bucal no cotidiano de idosos institucionalizados e não institucionalizados da cidade do Recife (PE, Brasil). Ciênc Saúde Coletiva 2010; 15:2955-64.

32. Martins AMEBL, Barreto SM, Silveira MF, SantaRosa TTA, Pereira RD. Autopercepção da saúde bucal entre idosos brasileiros. Rev Saúde Pública 2010; 44:912-22.

33. Organização Mundial da Saúde. Levantamentos básicos em saúde bucal. 4a Ed. São Paulo: Editora Santos; 1999.

34. Silva SRC. Autopercepção das condições bucais em pessoas com 60 anos e mais de idade [Tese de Doutorado]. São Paulo: Faculdade de Saúde Pública, Universidade de São Paulo; 1999.

35. Rodrigues CK. Autopercepção de saúde bucal em idosos [Dissertação de Mestrado]. Piracicaba: Universidade Estadual de Campinas, 2005.

36. Ferreira RC, Magalhães CS, Rocha ES, Schwambach CW, Moreira AN. Impacto psicossocial e funcional da condição de saúde bucal em idosos hospitalizados. RPG Rev Pós-Grad 2007; 14:279-85.

37. Atchison KA. The General Oral Health Assessment Index (GOHAI). In: Slade GD, editor. Measuring oral health and quality of life. Chapel Hill: University of North Carolina, Dental Ecology; 1997. p. 71-80.

38. Ministério da Saúde. Projeto SB2000: condições de saúde bucal da população brasileira no ano 2000. Manual do examinador. Brasília: Ministério da Saúde; 2001.

39. Associação Brasileira de Empresas de Pesquisas. Adoção do CCEB 2008. Critério de Classificação Econômica Brasil. http://www.abep.org.br (acessado em 07/Jul/2009).

40. Departamento de Atenção Básica, Secretaria de Atenção à Saúde, Ministério da Saúde. Histórico de cobertura - saúde da família. http://www.dab. saude.gov.br/historico_cobertura_sf.php (acessado em 07/Jul/2011).

41. Rosa AGF, Fernandez RSC, Pinto VG, Ramos LR. Condições de saúde bucal em pessoas de 60 anos ou mais no Município de São Paulo (Brasil). Rev Saúde Pública 1992; 26:155-60.

42. Silva SRC, Valsecki Júnior A. Avaliação das condições de saúde bucal dos idosos em um município brasileiro. Rev Panam Salud Pública 2000; 8:268-71.

43. Colussi CF, Freitas SFT, Calvo MCM. Perfil epidemiológico da cárie e do uso e necessidade de prótese na população idosa de Biguaçu, Santa Catarina. Rev Bras Epidemiol 2004; 7:88-97.

44. Konishi C, Hakuta C, Ueno M, Shinada K, Wright FAC, Kawaguchi Y. Factors associated with selfassessed oral health in the Japanese independent elderly. Gerodontology 2010; 27:53-61.

45. Atieh MA. Arabic version of the Geriatric Oral Health Assessment Index. Gerodontology 2008; 25:34-41.

Recebido em 27/Jan/2011

Versão final reapresentada em 27/Set/2011 Aprovado em 13/Fev/2012 